\title{
Computer Aided Evaluation of Aircraft Handling Qualities and

\author{
Flight Control System Robustness
}

Shyam Chet.ty and Raol J R

Flight Mechanics and Control Division

National Aeronautical Laboratory, Bangalore-17, India

\begin{abstract}
This paper describes the salient features of an interactive computer aided software package developed for the analytical evaluation of aircraft handling qualities and flight rontroi system robustness. The package is based on MATLAB/FORTRAN and runs on IBM compatible personal computers.
\end{abstract}

\section{Introduction}

Modern high performance aircraft are designed to be aerodynamically unstable and employ sophisticated digital flight control systems onboard, to recover stability and meet the stringent performance requirements over the entire flight envelope. Performance evaluation and verification of these adsanced flight control systems require analytical comparison of system characteristics to various military specifications which include flight control and handling quality specifications like the USAF MIL-F-9490D, MIL-F-8785C ${ }^{2}$ and the recent ML-STD-1797? In addition, to gaurantee flight safety, the flight control system designer must ensure sufficient robustness for the complete closed loop system against parameter variations, external disturbances and subsystem failures. These evaluations are becoming inoreasingly complex and difficult as the design trend towards increased control system integration, sophistication and coupling continues.

The present paper describes the salient features of the interactive computer aided software package developed for the analytical evaluation of handing qualities, generation of lower order equivalent system models, estimation of robustness margins and generation of ideal handling quality models for each phase of flight. The software package is based on Matlab/Fortran-77 and runs on IBH compatible personal computers. The paper also covers certain new features/techniques used for model order reduction, evaluation of closed loop handling quality criteria and control system robustness. Extensive use of vector optimisation techniques based on the minimisation of the Kreisselmejer's function $^{4,5}$ is made to offer greater flexibilty to the user during evaluation. Typical set of results are also presented to illustrate the capabilities of the software.

The software has been developed based on the popular MATLA8 (Moler etal., 1989) Computer Aided Control System Design (CACSO) tool. The software package has a modular structure consisting of 406 Matlab macros (.m files), 32 data files (, a at files) and 5 fortran executable code segments. Special effort has been taken to make the user interface uniform and consistent over the full scope of the package. A query/menu driven input has been used, as large number of parameters have to be entered by the user during evaluation. Extensive use of default values, on screen help messages, graphic outputs and a comprehensive demonstration programme provide the necessary support to a new user. The package has been split into the following sections which are selectable from the main menu and are classified as:

i Model Order Reduction Techniques

i Longitudinal Handling Quality Criteria iii Lateral/Directional Handling Quality Criteria

iv Demonstration Programmes

$\checkmark$ Ideal Handling Quality Design Models

yi Robustness Metrios

In each classification a specific criterion or technique can be selected from seperate sub menus which have been organised in a tree like structure. The package can handle multiple models (at different flight conditions) and these can be specified in either the state space or transfer function formats. Time or frequency response data from nonlinear simulation and flight tests can also be used to evaluate the aircraft performance. The final results, many of which are in 
graphical form, are stored in . HET files generated automatically using the Graphics Post Processor (OPP) option of MATLAB. On completion of an evaluation session, the user can select the print quality/format and output the the final results to a printer/plotter in a sequential manner to suit his particular application. Model Order Reduction Techniaues

The following model order reduction techniques are incorporated in the package for evaluating Lower Order Equivalent system (LOES) models :

i) Least Square Curve Fitting in the Frequency Domain - Levy's Technique and Modifications?, 8

ii) Scalar Optimisation Technique based on Rosenbrock's Search Routine 9

ifilstate Space Equivalent System Procedure $\mathbf{1 0}^{\mathbf{1 0}}$

iv) Vector Optimisation Technique based on Minimisation of the Kreisselfeier's Technique ${ }^{11}$

a) Longitudinal Dynamics (Second and Fourth Order Models)

b) Lateral/Directional Dynamics (First Second and Fourth Order Models with Extensions for Multiple Transfer Function Matching)

These techniques have been si ected based on the earlier experience of researchers in this area and gives the user the choice to select the technique best suited to his particular application. The user must select the initial values with care, as most of the above minimisation techniques will tend to converge at the local minima. Single step procedures like the least square curve fitting technique can be used to obtain the starting values prior to optimisation. The other important selection the user must make is the number of LOES parameters which are to be kept fixed during the minimisation process. This mainly depends on the structure of the control system and number of plant inputs. The software also incorporates several features which enables the user to overcome some of the problems commonly encountered, like non uniqueness of solutions, interpretation of matching cost, goodness of fit required etc. Figure 1 shows the bounds on amplitude and phase error for the pitch rate transfer function as a function of frequency to which the pilot is insensitive ${ }^{9}$. The vector optimisation procedure for model reduction permits these error bounds to be expilcitly included as constrints, For multiple transfer function matching the technique allows the user to minimise simultaneously the errors in each of the transfer functions. Figure 2 shows the results obtained using the lateral/directional model order reduction software for a highly augmented aircraft model.

\section{Longitudinal Handling Qualities}

The requirement guidance section of the MIL-STD-1797 lists several alternative short period handling quality criteria that needs to be evaluated for modern high performance aircraft. Each criterion can be selected from appropriate sub-menus by the user. Tables - 1,2 gives the details of a typical menu for longitudinal handling qualities. Figures 3 to 8 show the results obtained. The integer numbers ( 1 to 7 ) in the plots indicate the results at different flight conditions for which the evaluation was done.

\section{Lateral/Directional Handling Qualities}

The different lateral/direotional criteria that can be evaluated using the software package are listed in Table 3. Typical results are shown in figures 9 to 11.

Generation of Ideal Handling Quality Models

Most modern control design approaches require the designer to formulate an "ideal model" prior to starting the actual design process. For high performanoe aircraft, formulation of these low order models are crucial, and due to the large number of specifications and practical constraints which the designer must meet, this is an extremely complex task. This package allows the user to generate such models based on the airframe characteristics, flight condition and particular task. The procedure adopted for generating the optimal plant matrices is based on the simultaneous solution of a set of' independent nonlinear equations. The desired specifications (including handling qualities) are formulated as a number of independent nonlinear equations containing the dimensional derivatives as unknowns which are solved simultaneously using a routine based on the secant method for finding the roots of an equation. The end 
results depend on the choice of the initial values for these unknowns (dimensional derivatives) and must be systematically selected. To ensure good convergence the procedure allows the user to start with a reduced set of equations and once valid solutions are found, additional equations are added one or two at a time until all the unknotrs are included. Typical specifications used are expressions for the demping/natural frequencies for the dominant modes, modal phase angles between motion variables at the appropriate frequencies and gain ratios ${ }^{12}$. An ideal state space model which meets all the specifications listed in the MIL-STD-1797 for a typical high performance aircraft flying at low speed and low altitude is given in Table 4. Figure 12 shows the short and long term time responses for a step input to the ideal model.

\section{Robustness Analysis}

Design of a control system is done with a nominal model of the plant. However, the control system characteristics do not remain the same in presence of uncertainties. These uncertainties are due to variations in parameters ${ }^{13,14}$ and /or ungodelled or incorrectly modelled dynamics One of the main aims of control system synthesis is to achieve stability and good performance in face of model uncertainties. Such issues are treated via robustness analysis.

We describe some methods and results of robustness analysis. Specifically the approaches based on i) singular values and eigenvalues of return and inverse return difference matrices, ii) multivariable gain and phase margins, iii, single-loop-at-stime analysis (LAATA) and iv modal analysis. The numerical results are presented where appropriate.

\section{Robustness Problem}

When a compensator is designed us nz a nominal model of the plant (e, $\mathbf{s}$ : aircraft), the resulting feedback control system (FCS) is said to be robust with respect to class of modelling errors if it remains stable and has good performance, when the nominal model is replaced by any other model of the plant. We discuss only stability robustness Gain /Phase Harkin issues

In a practical design it is necessary to provide reasonable stability margins, 1 : e, to provide sufficient gain and phase margins (GMPH), For a single-input-singleoutput (SISO) system GMpMs are directly related to the variation, of the so called return difference quantity $\{\mathrm{RD} \ldots\rangle \mathrm{T}\langle s\rangle=$ $1+H(s) G(s)$, with trequency. The RD plays important role in the assessment of the robustness of a control system. The issue in oharacterising stability margin for the FCS is to determine a lower bound on the size of the smallest perturbation to the $\mathrm{RD}$ matrix (for aultivariable systems, MVS) that will destabilize the system. It is assumed that the uncertainty introduced in the flight control system from various sources can be represented as a gain and/o phase uncertainty in each feedback loop. Therefore, the stability margin requirements of the flight control specifications Yetermine the largest admissilile gain and phase uncertainties in each loop. For $\mathbf{F C s}^{\mathbf{1 5}}$, the requirement is - In multivariable system, variations shall be made with all gain and phase values in the feedback paths held at nominal values except for the path under investigation. More meaningful stability, margins may be defined as limits within which the gains of all feedback loops may vary independently at the same time without destabilizing the system, while the phase angles remain at their nominal values. This concept also applies to phase variations while gains remain at their nominal values. This amounts to setting the limits for independent gain and phase variations in a diagonal perturbation matrix for a multiplicative perturbation model. We can also define another approach for uniform variation of gains and phases.

Stability Margins for Multivariable Systems

We explore two approaches: eigenvalue-based and singular value-based methods to arrive at gain/phase margin limits based on Ref. $[14]$.

Independent Gain/Phase Margins (IGHs, IPHs):

The independent gain (phase) margins are limits within which the gains (phases) of feedback loops may vary independently at the same time without destabilizing the system, while the phase angles ( gains) remain at their nominal values.

Let $L(s)$ be a diagonal perturbation matrix

$\left.L(s)=\operatorname{diag} \mid b_{1}(\omega) e^{j \theta_{1}(\omega)} \ldots b_{n}^{j \theta_{n}(\omega)}\right)$. 
Then IOMs are limits within which

$b_{i}(w)$ may vary independently for each $i$ without destabilizing the system, with $\theta .(\omega)=0 . \forall \omega$ and $\forall$ i. Similarly IPHs are limits within which $\theta .(\omega)$ may vary independently for each $i$ without destabilizing the system while $b_{i}(\omega)=1, v$ and $v \mathbf{i}$.

Uniform Gain/Ehase Margins (JGMs, UPHs):

The uniform gain (chase) margins are limits within which the gains (phase angles) of all feedback loops vary uniformly (as against independent ones) at the same tine without destabilizing the system, while the phase angles (gains) remain at their nominal values.

In this case $\mathbf{L}(j \omega)=\mathbf{b}(\boldsymbol{j}) \mathrm{e}^{\mathbf{j \theta ( \omega )}} \mathbf{K}(\mathbf{j} \omega)$ and $K$ is a diagonal nominal complex loop gain matrix.

Thus UoMs wrt the nominal loop gain $K(j \omega)$ are limits within which b(j) may vary without, destabilizing the system while $\theta(\omega)$ $=0, \mathbf{v}$.

Similarly UPMs wrt the nominal loop gain are limits within which $\theta(\omega)$ may vary without destabilizing the feedback system while $b(\boldsymbol{s})=1$, $\boldsymbol{\psi} \boldsymbol{\omega}$. Based on the above definitions the robustness criteria arrived at by multivariable Nyquist theory have been implemented in this package. ${ }^{14}$

The combined use of IGMs, Uoms and IPHs, UPYs in an appropriate way obtains the extended stability regions beyond the usual conservative regions. However in the present case, only independent gain and phase margins have been used for seeking the stability regions.

\section{Loop-at-a-time Approach}

The single loop stability margins required in MIL-F-9490D are computed by manipulating the frequency response data base that is generated during the multivariable study of robustness. The approach is to obtain equivalent open loop transfer function for the loop in question by closing all the other loops in a MVS via a connection matrix: $\mathbf{K}_{\mathbf{c}}: \operatorname{diag}(1,1,1,1,0,1,1,1 \ldots\}$. Here ' $O$ ' in the diagonal implies that, that loop is under investigation to evaluate the stability margins, whereas the '1s' in the diagonal imply the loop closure for the remaining loops. With this connection matrix the equivalent (open) loop transfer function is evaluated for which the frequency response data base is used to compute the ith loop frequency response as:

$\mathbf{g}_{k}\left(j \omega_{i}\right)=\left\{G\left(j \omega_{.}\right)\left\{I+k_{c} G\left(j \omega_{i}\right)\right\}^{-1}\right.$ Here $(.)_{k k}$ means the $(k, k)$ th element of the matrix. The single loop transfer function is then used to define $a_{1}$ and $a_{2}$, namely :

$$
\begin{aligned}
& a_{1}=\min _{\omega \geqslant 0} \overline{E\left[I\left[t s_{k}^{-1}(j \omega)\right\}^{-1}\right]} \\
& =\operatorname{nin} \mid 1+1 / \mathbf{g}_{\mathbf{k}}(\mathbf{j}(\boldsymbol{)}) \mid \\
& \omega \geqslant 0
\end{aligned}
$$

similarly

$$
\begin{aligned}
& \mathbf{a}_{\mathbf{2}}=\operatorname{ain} \\
& \omega\rangle_{\wedge} 0 F\left[1 I+g_{k}(j \omega)\right\}^{-1} I \\
& =\min \mid l+z_{k}(j(\alpha) \mid
\end{aligned}
$$$$
\omega \geqslant 0
$$

Then the gain and phase margin formulae are applied to $a_{1}$ and $a_{2}$ to obtain the gain and phase margins for the feedback loop in question, that is the loop for which these margins are to be evaluated. This procedure is then applied in turn to each feedback loop to obtain margins for these loops. The approach is analytically complementary to the Bode diagram method to obtain gain and phase margins and it can be used also for nonminimum phase loops without any modifications. If margins estimated by this method are greater than specified by MIL-F-9490D, then actual margins are also expected to be greater.

Modal Analysis/Closed Loop Eigenvalue Looj

In this approach the method described in Ref. $\mathbf{1 7}$ is implemented. Let the aircraft dynamics be represented as

$$
\begin{aligned}
& \dot{x}=\mathrm{Ax}+\mathrm{Bu} \\
& \mathrm{y}=\mathrm{Cx}+\mathrm{Du}
\end{aligned}
$$

Then output feedback is given as:

$$
u=\overline{\mathbf{k}} \mathbf{y}+\mathbf{u}_{\mathrm{P}}
$$

Here $L_{P}$ is an external reference input and other variables have standard state space notation and interpretations.

In eigensystem assignment, the problem is to select a $\widetilde{x}$, the output feedback gain matrix, such that the closed loop system has desired eigenvalus/eigerveotors specifications (eigen characteristics). 
The closed loop s,rstew atrix then becomes $A_{C l}=A+B \hat{k} C$

where

$$
\hat{\mathbf{k}}=P \widetilde{\mathbf{K}} \text { and } P=\left[\left[_{\mathbf{n}}-\widetilde{K} 0\right]^{-1}\right.
$$

A modal matrix is defined as

$$
T=\left[v_{1}, v_{2}, \ldots, v_{n} 1\right.
$$

uherin

$$
v_{i} \text { are the }
$$

eigenvectors

corresponding to eigenvalues $\lambda_{1}$ of (open or closed loop, system. In the present analysis determinant and condition number (cond ( $T$ )) of a modal matrix are evaluated for various case study examples.

Reduction in condition number of (closed loop) modal matrix indicates improved design of the FCS. Sriother useful method of studying the stability properties of control system is root locus, whloh portrays the movement of closed loop poles of a system as function of (open) loop gain. A slight variation of the above approach is chosen for analysis of the FCS. At each iteration, the closed loop eigenvalues are obtained, which reflect the effect of perturbation gains (matrix $\mathbb{X}$ ) in the loop transfer function.

Case Study I :

Lateral Attitude Control System of a DRONE Aircraft.

a) Multivariable Results:

The perturbation matrix $L(j \omega)$ is

characterized by $L(j \omega)=\operatorname{dis}\left(\boldsymbol{\beta}_{\mathbf{1}} e^{j \beta 1}, \boldsymbol{\beta}_{\mathbf{2}}\right.$

$e^{j \theta 2}$, where $\beta$ and $\theta$ are constants in the gain and phase margin calculations. In this case (open) loop transfer function is a two input two output system and hence has two perturbation gains.

The regions of gain stability margins are shown in Fig. 13 for direction 1,2,3. In exploring the stability regions, for gain space, either the migration of closed loop eigenvalues to the right half of s-plane or the increase of minimum singular value after some iterations can be taken ss a stopping criterion. Due to numerical reasons the minimum singular value may not reach the zero value and it might either start increasing or remain almost steady. These conditions are observed in many situations and correlate consistently with behavior of closed loop eigenvalues loci, which either gradually converge to jw axis or cross and enter into the right half s-plane, thereby indicating that the stability boundary has been nearly reached, The phase margin stability regions for direction 1,2 and 3 are shown in Fig. 14. It was found that stability boundary is reached (in corresponding direction) at iteration number that corresponds to either start of monotonous increase in singular value or to a start of very slow decresse in singular value as function of iteration number.

The matching of sain/phase margin stability regions as shown in 8 is 13,14 with those of Ref. 14 (in the relevant directions) is in close agreement. It is a matter of (computer) time and patience that decide the explorations in all remaining directions, if need be so. It is possible to fully automate the prooedure by incorporating the exploration of those stability boundaries using eigenvalue based uniform gain and phase margins. The rectangular boxes must be assumed to be closed and stable combination of gain/phsse values lies within as well on the boundaries of these boxes.

b) Loop-at-a-time Results:

The gain and phase margins obtained are given as:

Loop $1 \mathrm{GM}=t /-25.82 \mathrm{~d} ; \mathrm{gH}=t /-63.42 \mathrm{dep}$. Loop $2 \mathrm{G}$ 月 $=t /-4.59 \mathrm{~dB} ; \quad P H=\$ /-40.63 \mathrm{deg}$. which seem to meet the MIL-F-9490D specifications for gain and phase margins.

Fig. 15 depicts results of loop-at-a-time analysis. It can be seen from the figure that the gain and phase margins estimated by analytical method have conservative values.

o) Modal Analysis Results

\begin{tabular}{lc}
\hline OL Eigenvalues & CL Eigenvalues \\
\hline-20.0000 & -20.1481 \\
-20.0000 & -18.6991 \\
-0.0370 & -0.6909 \\
-3.2497 & -2.5919 \\
$0.1900+/-1.0507 i$ & -2.2703 \\
& $-1.1870 i$ \\
\hline Det (T) 5.6871 e-05 & $1.1255 \mathrm{e}-08$ \\
Cond (T) 62.7312 & 822.6957 \\
\hline
\end{tabular}

\{ here $T$ is a modal matrix \}

Case Study II :

Lateral Flight Control System of an Advanced Fighter Aircraft. 16

a) Multivariable Results:

The gain margin stability regions for 
directions 2,3 and 4 are shown in Fig. 16. The stability boundaries are reached, at iteration 15 for direction 2, and at iteration 4 or 5 for directions 3 and 4 in gain-plane region of stability. This is supported by the behaviour of minimum singular value and the closed loop eigenvalues. 13

b) Loop-at-a-time Results:

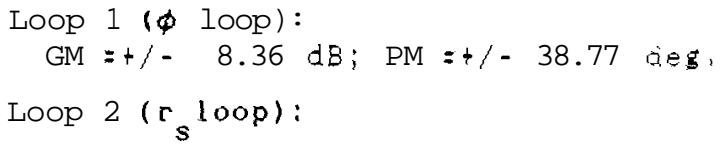

Comparison of the above values with those of MIL-F-9490D values, shows that margins are satisfactory. However, the analytically predicted values are found to be conservative.

Case Study II I:

a) Multivariable Results: The gain-plane region of certain flight condition 17. It can be easily reached at iteration 4 . b) Loop-at-a-time Results: Cain/Phase Margins are:

\begin{tabular}{llrl}
\hline Loop Margin & FC A & FC B \\
(Land/Appoh.) & (HIGH a) \\
\hline 1 & GM & 5.75 & 6.13 \\
& PM & 55.80 & 32.08 \\
2 & GM & 21.02 & 21.82 \\
& PM & 59.21 & 58.74
\end{tabular}

For flight conditions and loops shown, the MIL-F-9490D is clearly satisfied.

c) Modal Analysis Results:

The results obtained using this package agree with those of Ref. 17. Detailed results of all the case studies of robustness analysis are given in Ref. 13.

\section{Conclusions}

In this paper the development of a computer aided software package and it validation for evaluation of handling quality criteria and robustness analysis are described. The interactive PC MATLAB/F-77 based package is validated for several flight control systems available in open literature.

\section{References}

1. Anonymous, Military Specifications for Flight Control Systems - Design Installation and Test of Piloted Aircraft, MILF-9490D(USAF), 1975.

2. Anonymous, Military Specifications for Flying Qualities of Piloted Airplanes, MIL-F-8785C(USAF), 1980.

3. 800z, J.E., "Relative Evaluation of MILSTD-1797A, Longitudinal Flying Qualities Applicable to Flared Landing and Approach", AIAA Paper 88-4363, 1988.

4. Kreisselmeier, G. and Steinhauser, $R$, , "Systematic Control Design by optising Vector Performance In ex" : IFAC syoosium on Computer Aided Design of Control Systems, Zurich, pp. 13-117, 1979.

5. Chetty, S., and Usha, U.N.," "Computer Aided Handling Qualit es Evaluation in the Frequency Domain ", NAL-TM-SE-8902, India, 1989.

6. Moler, C., Little, J. Bangert, $S$, "PCMATLAB User's Guide", Mathworks Inc., MA -01770, U.S.A., 3.5g Edition, 1989.

7. Saraswathi, L., and Chetty, S., Longitudinal Equivalent Systems for Augmented Airoraft using Least Squares Curve Fitting Techniques", NAL PD \$C 9011, Deo, 1990.

8. Ismail, S., and Chetty, S., "Lower Order Equivalent Systems for High Performance Aircraft", AMSE International Conference on Signals, Data and Systems SDS-91, New Delhi, India, Dec, 1991.

9. Moorhouse, D.J. and Woodcock, R,J, " Background Information and User's Guide for MIL-F-8785C, Military speoification for Flying Qualities of Piloted Airplanes", AFWAL-TR-81-3109, 1981.

10. Bacon, B.J. and Schmidt, D.K., "Fundamental Approach to Equivalent Systems Analysis", AIAA JI. of Guidance, Vol. 11, No. 6, pp. 527-534, 1988.

11. Chetty, S., and Lakrhmi, P.," Computer Aided Evaluation of Aircraft Handling Qualities", 5th IFAC/IMACS Symposium on Computer Aided Design in Control Systems, Syansea, U,K, July 1991.

12. MoRuer, D., Ashkenas, I., Graham, D., "Aicraft Dynamics \& Automatic Control", Princeton Uniy. Press, N.J., 1974.

13. Raol, J. R.," Robustness Analysis of Flight Control Systems-based on Singular Values/Eigenvalues", NAL PD FC 9109.

14, Ridgely, D.B., and Banda, S.S., "Introduction to Robust Multivariable Control", AFWAL-TR-85-3102, Feb, 1986.

15. Anderson, M.R., Rubin, U.H., Vincent, J.H., "Evaluation Methods for Complex Flight Control Systems", AIAA c8-89- 
$3502,1989$.

16. MoRuer, D.T., Myers, T.T., Thompson, P.M.,"Literal Singular Value-based Flight Control System Design Techniques", AIAA J1, of Guidance and cont., Vol. 12, No.6, Nov-Dec, 1989

17. Srinathkumar, $S$, "Modal Control Theory and Application to Airoraft Lateral Handling Qualities Design", NASA Technical Paper 1234 , June 1978.

\section{Achnowledgements}

Authors are grateful to Dr. Balakrishna $S$. (former Head, FMCD) and Dr. Srinathkumar $S$. Head, FMCD, for their technical guidance leading to thought provoking discussions during the course of this work. Also the contributions of Ms. P. Lakshai, Mr. Shaik Ismail and Mrs. Saraswathi L. towards the development of this software are highly appreciated

Table 1 Longitudinal Handling Qualities

1. Equivalent Systems

2. Control Anticipation Parameter (CAP)

3. Bandwidth Criterion

4. Neal-Smith Criterion

5. Closedloop Criterion (MIL-STD-1797A)

6. Gibsun's Criteria

7. Pitch Rate Response Criterion

a. C* Criterion

9. Time Responses (Step/Inpulse Input)

10. Turbulence Response (Random/Disorete)

11. Phugoid Damping

Table 2 Gibson's Critera

1. Q-Theta Trends

2. $\mathrm{Nz}$ Criterion

3. PIO Criterion (Up and Away)

4. Pitch Angle Criterion (Approach and Landing)
Table 3 Lateral/Directional HQ's

1. Equivalent Systems

2. IPhi/Betaid Computation

3. Lateral/Directional Mode Characteristics

4. Roll Rate Oscillations

5. Roll Performance

6. Bank Angle Oscillations

7. Sideslip Excursions

8. Time Responses (Step/Pulse Inputs)

9. Turbulence/qust Response

Table 4 Ideal HQ Model

Openloop Aircraft State Space Model :

FC - 0.5 Mach, $h=5000 \mathrm{~m}$, Level Flight

$X=\{a \mid f a, q$, thet $a, u / v 0\}, v=Y=[a z]$

$$
\begin{aligned}
& : \begin{array}{llll}
-0.648 & 1.000 & 0.000 & -0.127
\end{array} \\
& \text { [A] }=\mathbf{i} \begin{array}{llll}
0.452 & -0.996 & 0.000 & -0.025
\end{array} \\
& \text { : } 0.000 \quad 1.000 \quad 0.000 \quad 0.000 \text { : }
\end{aligned}
$$

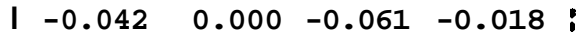

$$
\begin{aligned}
& :-0.287: \\
& {[B]=-15.340:} \\
& \begin{array}{rr}
\quad 0.000 \\
-0.027
\end{array} \\
& {[C]=\mid-104.110 .000 \quad 0.000-20.586} \\
& {[D]=:-46.424 ;}
\end{aligned}
$$

Ideal State Space Model :

$\begin{aligned} & {[A i]=} \begin{array}{rrrr}-0.726 & 1.000 & 0.000 & -0.210 \\ -3.249 & -3.002 & 0.000 & -0.034 \\ 0.000 & 1.000 & 0.000 & 0.000 \\ 0.068 & 0.000 & -0.061 & -0.014\end{array} \\ &\{B i]=[B] ; \quad[C i]=[C] ;[D i]=[D]\end{aligned}$



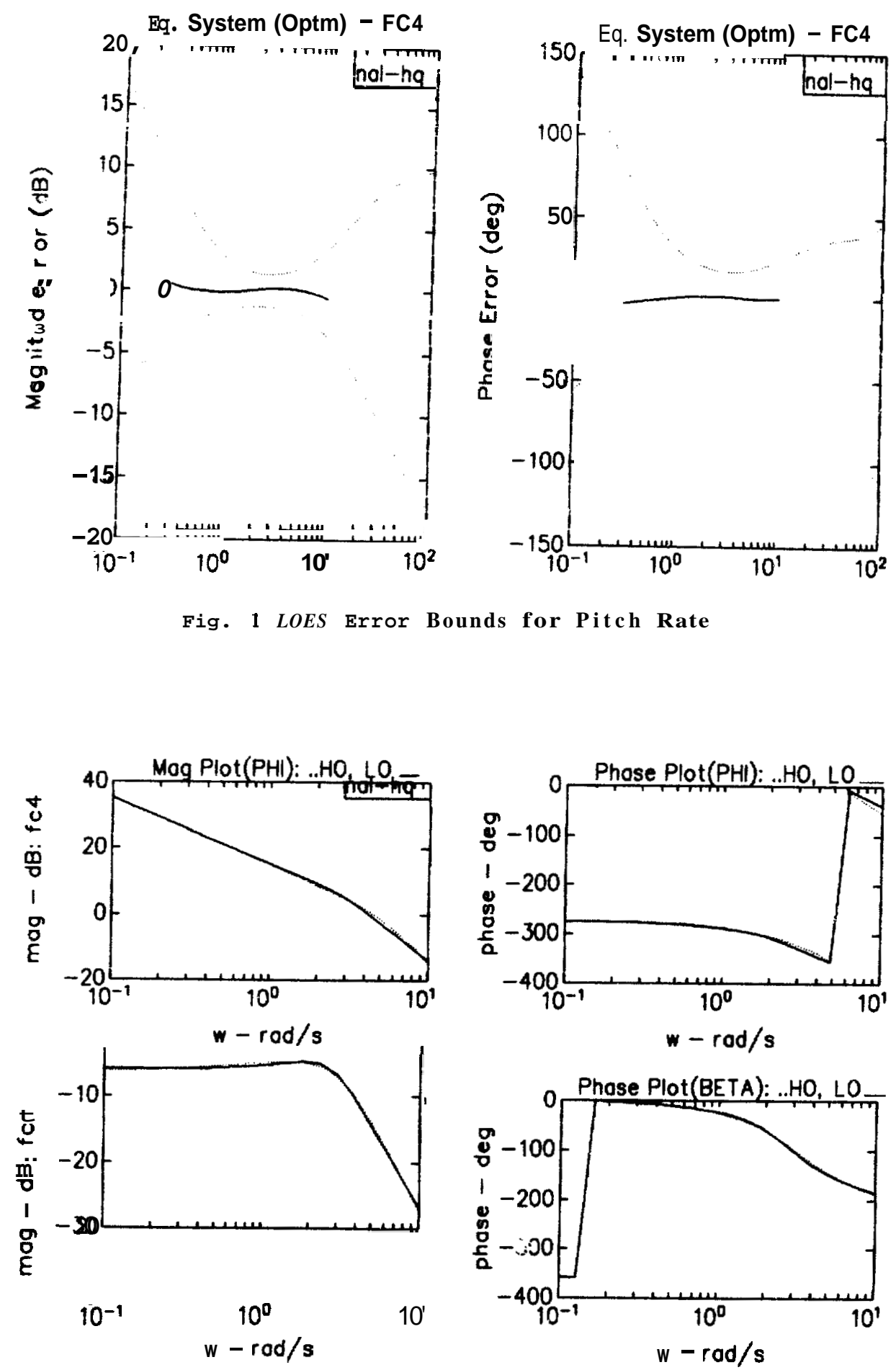

Fig. 2 Frequency Response Comparison for Lateral/Directional Axes 


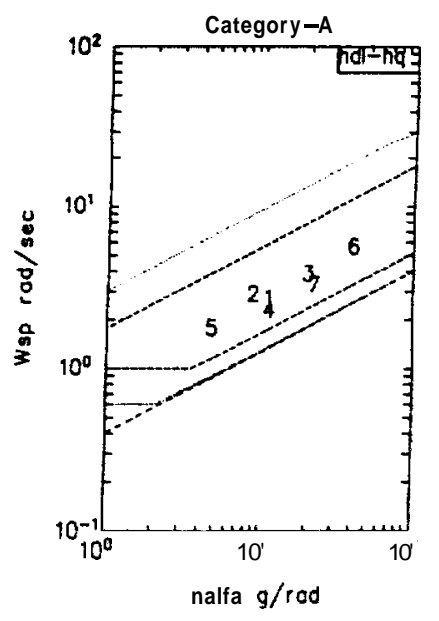

Fig. 3 Short Period Frequency Bounds

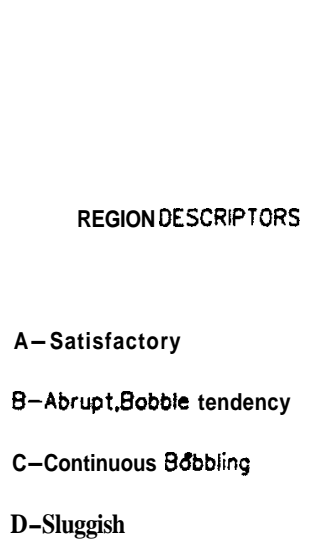

Fig. 5 Gibson's Q-Theta Trends

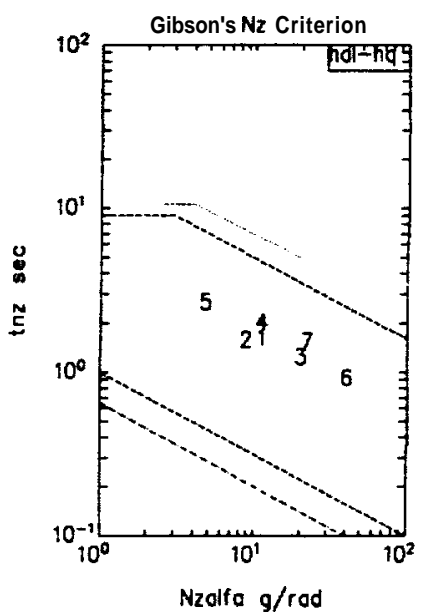

Fig. 7 Gibson's Nz Criterion

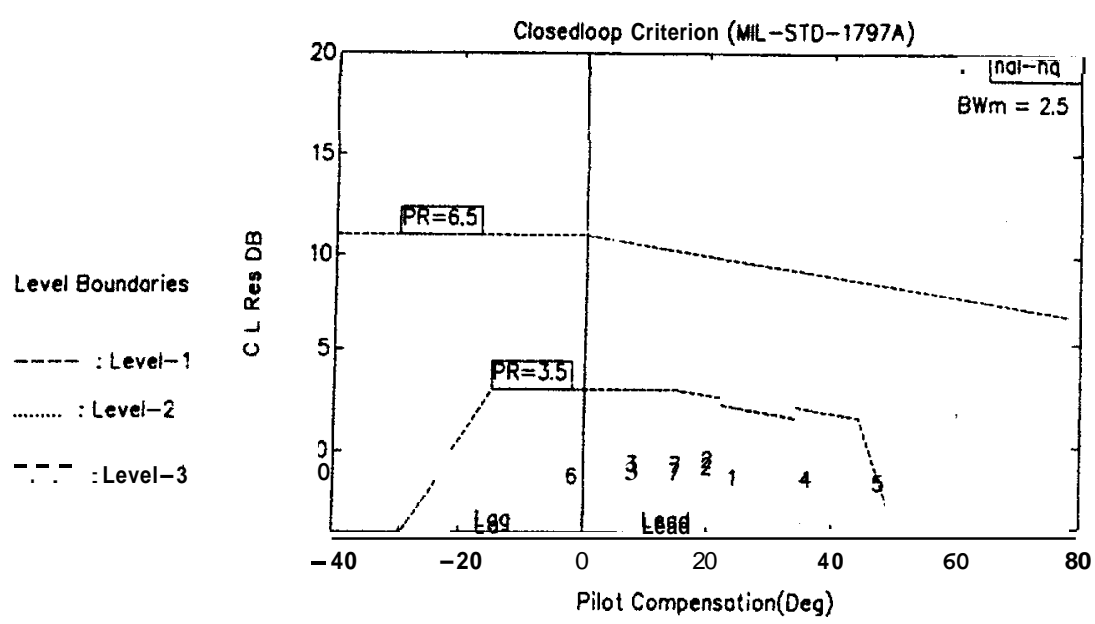

Fig. 4 Closedloop Criterion (Neal-Smith)

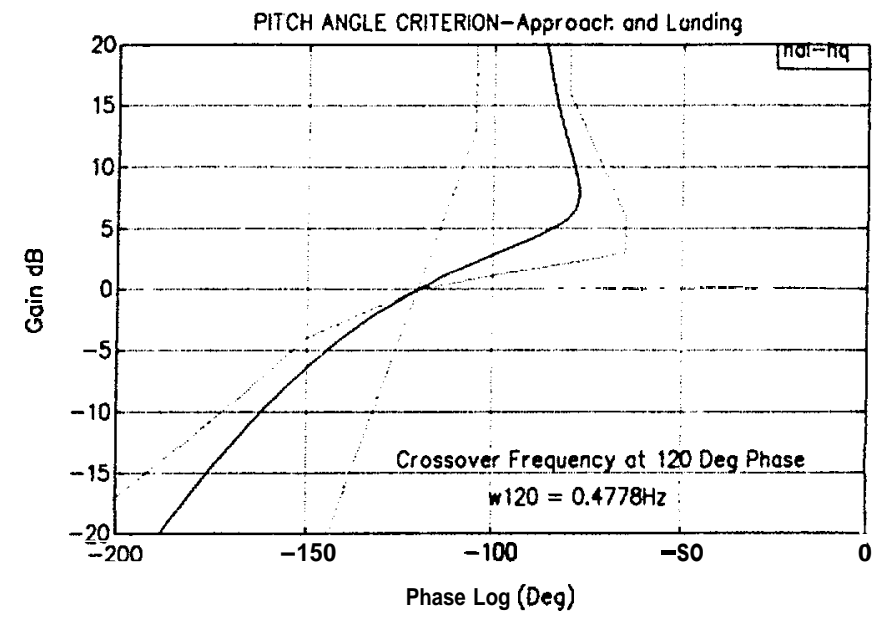

Fig. 6 Gibson's Pitch Angle Criterion

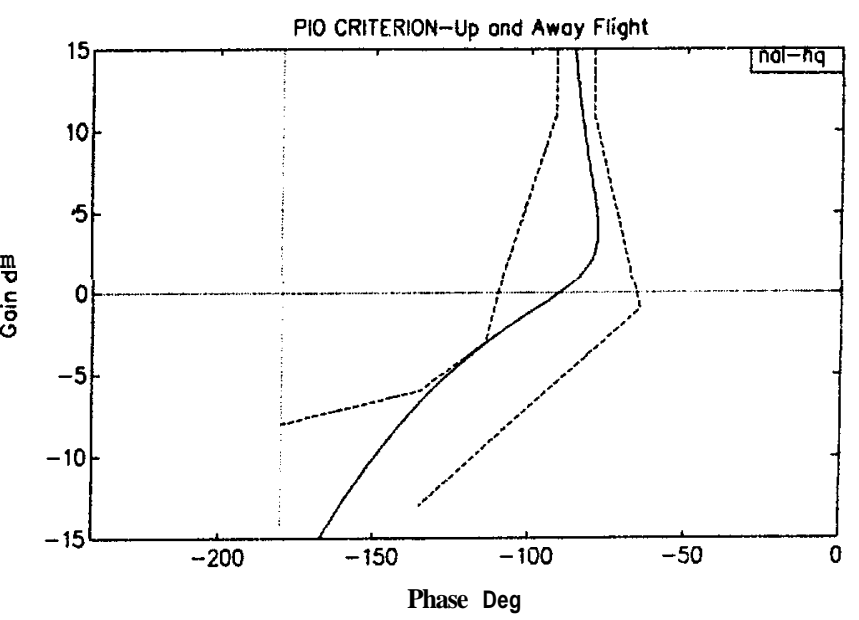

Fig. 8 Gibson's PIO Criterion 


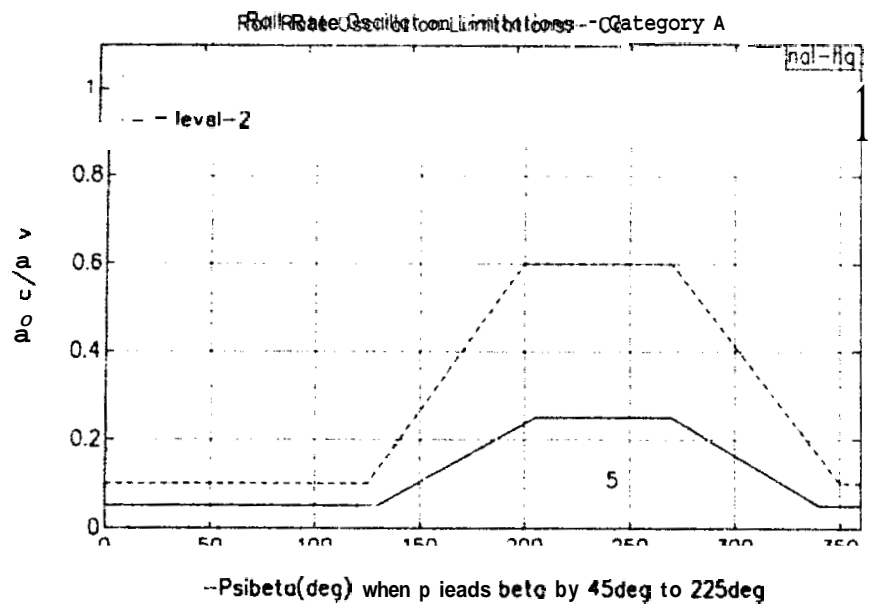

Fig. 9 Roll Rate Oscillation Criterion

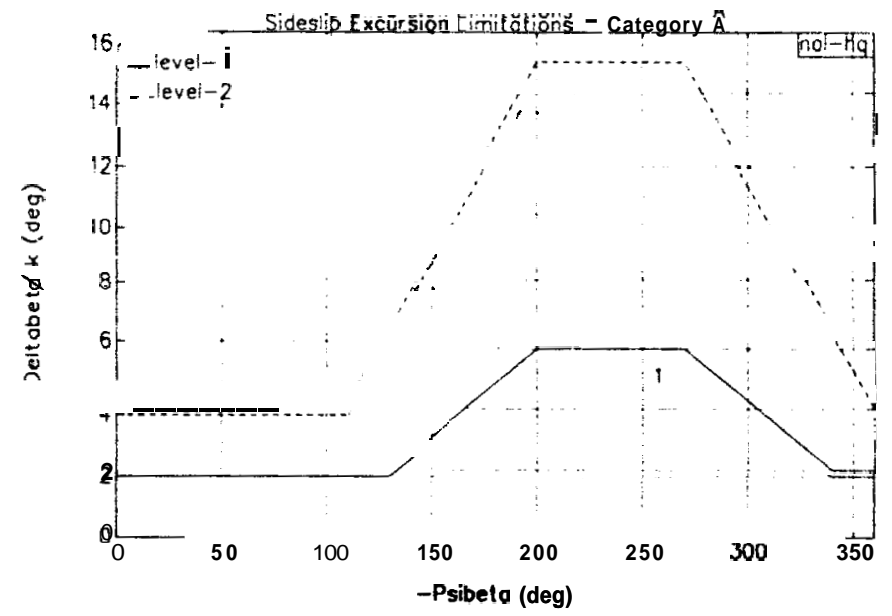

Fig. 10 Sideslip Excursion Limits

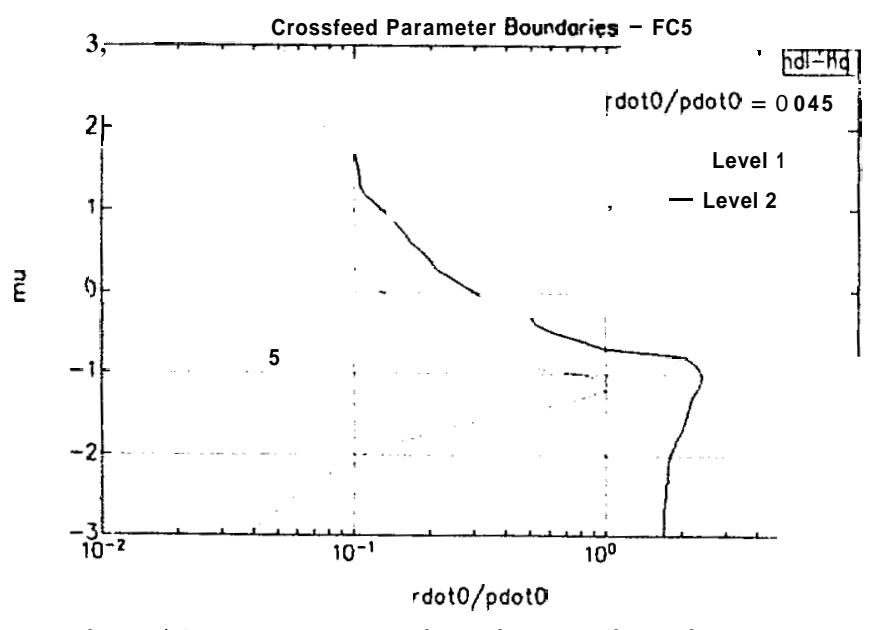

Fig. 11 Turn Co-ordination Criterion
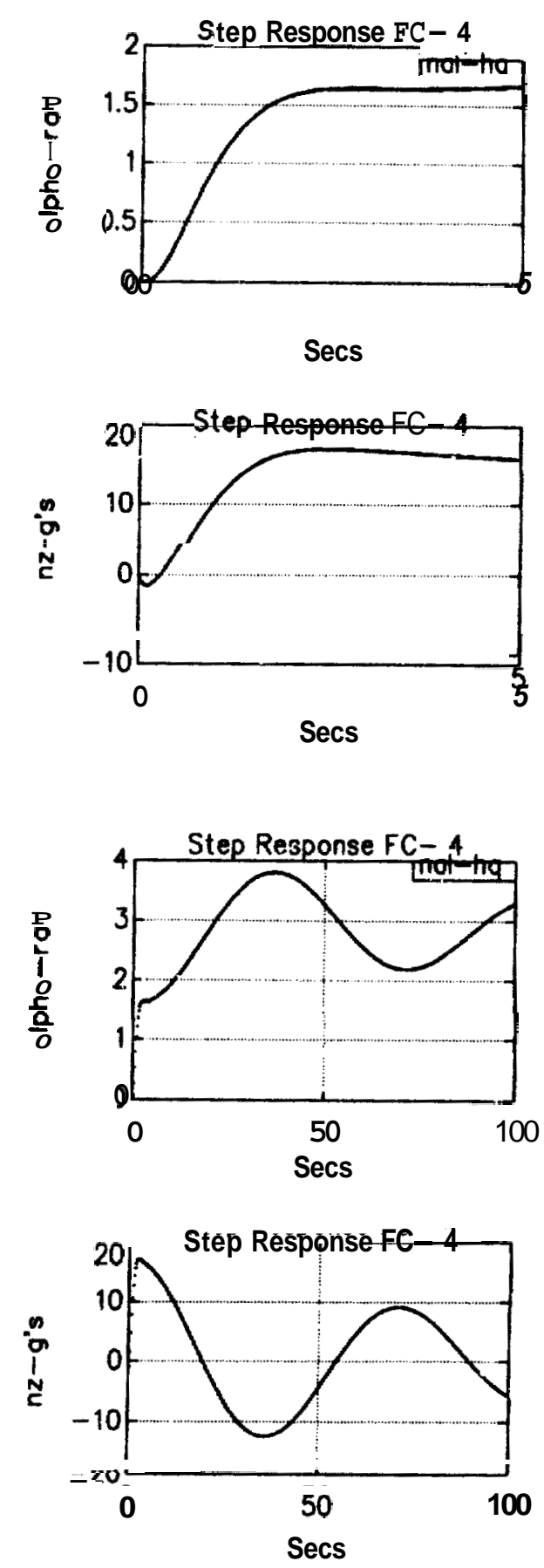

Fig. 12 Ideal HQ Model Step Responses (Short and Longterm) 

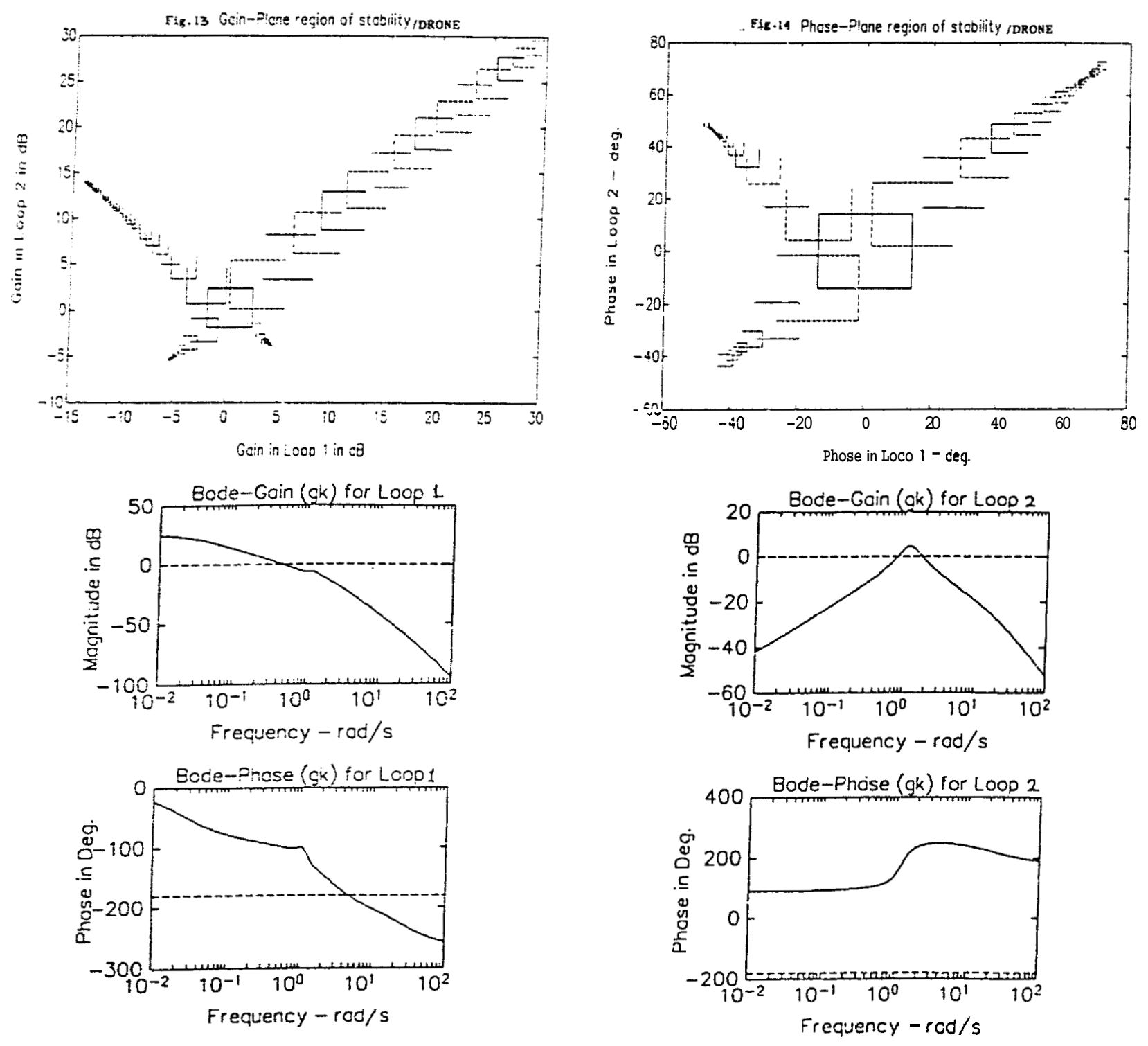

Fig. 15 Loop-a -a-tine Results for Case I
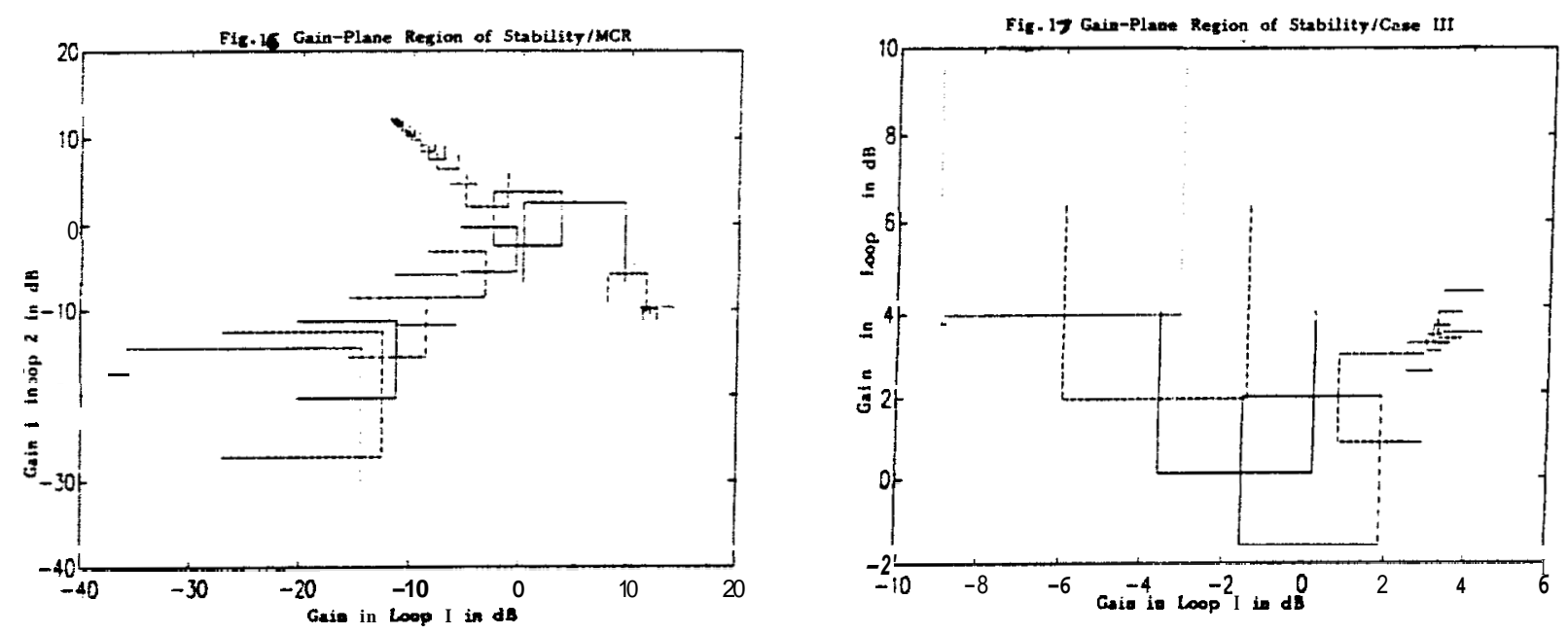


\section{Computer Aided Evaluation of Aircraft Handling Qualities and

\author{
Flight Control System Robustness
}

Shyam Chet.ty and Raol J R

Flight Mechanics and Control Division

National Aeronautical Laboratory, Bangalore-17, India

\begin{abstract}
This paper describes the salient features of an interactive computer aided software package developed for the analytical evaluation of aircraft handling qualities and flight rontroi system robustness. The package is based on MATLAB/FORTRAN and runs on IBM compatible personal computers.
\end{abstract}

\section{Introduction}

Modern high performance aircraft are designed to be aerodynamically unstable and employ sophisticated digital flight control systems onboard, to recover stability and meet the stringent performance requirements over the entire flight envelope. Performance evaluation and verification of these adsanced flight control systems require analytical comparison of system characteristics to various military specifications which include flight control and handling quality specifications like the USAF MIL-F-9490D, MIL-F-8785C ${ }^{2}$ and the recent ML-STD-1797? In addition, to gaurantee flight safety, the flight control system designer must ensure sufficient robustness for the complete closed loop system against parameter variations, external disturbances and subsystem failures. These evaluations are becoming inoreasingly complex and difficult as the design trend towards increased control system integration, sophistication and coupling continues.

The present paper describes the salient features of the interactive computer aided software package developed for the analytical evaluation of handing qualities, generation of lower order equivalent system models, estimation of robustness margins and generation of ideal handling quality models for each phase of flight. The software package is based on Matlab/Fortran-77 and runs on IBH compatible personal computers. The paper also covers certain new features/techniques used for model order reduction, evaluation of closed loop handling quality criteria and control system robustness. Extensive use of vector optimisation techniques based on the minimisation of the Kreisselmejer's function $^{4,5}$ is made to offer greater flexibilty to the user during evaluation. Typical set of results are also presented to illustrate the capabilities of the software.

The software has been developed based on the popular MATLA8 (Moler etal., 1989) Computer Aided Control System Design (CACSO) tool. The software package has a modular structure consisting of 406 Matlab macros (.m files), 32 data files (, a at files) and 5 fortran executable code segments. Special effort has been taken to make the user interface uniform and consistent over the full scope of the package. A query/menu driven input has been used, as large number of parameters have to be entered by the user during evaluation. Extensive use of default values, on screen help messages, graphic outputs and a comprehensive demonstration programme provide the necessary support to a new user. The package has been split into the following sections which are selectable from the main menu and are classified as:

i Model Order Reduction Techniques

i Longitudinal Handling Quality Criteria iii Lateral/Directional Handling Quality Criteria

iv Demonstration Programmes

$\checkmark$ Ideal Handling Quality Design Models

yi Robustness Metrios

In each classification a specific criterion or technique can be selected from seperate sub menus which have been organised in a tree like structure. The package can handle multiple models (at different flight conditions) and these can be specified in either the state space or transfer function formats. Time or frequency response data from nonlinear simulation and flight tests can also be used to evaluate the aircraft performance. The final results, many of which are in 